\title{
Can mini-tracheostomy needle be safer for residency training in percutaneous dilatation tracheostomy applications in intensive
} care unit?

\author{
@Mustafa Özgür Cırık, @Güler Eraslan Doğanay, @Ali Alagöz, @Hilal Sazak \\ University of Health Sciences, Atatürk Chest Diseases and Thoracic Surgery Training and Research Hospital, Department of Anesthesiology \\ and Reanimation Turkey
}

Cite this article as: Cırık MÖ, Eraslan DOğanay G, Alagöz A, Sazak H. Can mini-tracheostomy needle be safer for residency training in percutaneous dilatation tracheostomy applications in intensive care unit?. J Health Sci Med 2021; 4(2): 228-232.

\begin{abstract}
Aim: The aim of this study is to evaluate the bedside percutaneous dilatation tracheostomy (PDT), performed with fiberoptic bronchoscopy guided Griggs technique using a mini-tracheostomy needle, by residents in intensive care unit (ICU) retrospectively.

Material and Method: Twenty PDT applications were performed in ICU using mini-tracheostomy needle in the present study. All PDT procedures were performed by a resident who was currently undergoing ICU residency training. Griggs technique was used in all procedures. All complications, the time from needle insertion to the insertion of the tracheostomy cannula was also noted.

Results: The average age of the patients was 69.8 \pm 16.14 years. The mean Acute Physiology and Chronic Health Assessment (APACHE) II score of the patients was 23.05 \pm 6.16 , Glasgow Coma Scale (GCS) score was 10 \pm 3.43 , and Sepsis-Related Organ Failure Assessment (SOFA) score was 7.2 \pm 2.11 . The mean procedure time was $13 \pm 1.68$ minutes, and the day of tracheostomy application was $6.35 \pm 4.59$. Twelve $(60 \%)$ patients were switched to home-type ventilators. Ten $(50 \%)$ of the patients were transferred to the palliative ward with home-type ventilator. Mortality rate was $20 \%$ (4 patients) at 90 days. When patients were evaluated in terms of complications; none of the patients had pneumothorax, subcutaneous emphysema, posterior tracheal wall damage, or tracheoesophageal fistula. Minimal bleeding that required no intervention was observed in only one patient.

Conclusion: Using mini-tracheostomy needle in PDTs performed via fiberoptic bronchoscopy by less experienced residents may be safer to prevent complications.
\end{abstract}

Keywords: Mini-tracheostomy, PDT, residency training, mini-tracheostomy needle

\section{INTRODUCTION}

Recently, the percutaneous dilatational tracheostomy (PDT) technique, which is used in intensive care unit (ICU), in patients who require long-term mechanical ventilation (MV), has been used increasingly and with a higher rate compared to the surgical technique (1). PDT has some advantages such as easy application with a small skin incision at the bedside, less tissue damage, and limited complications $(2,3)$. It is possible to protect the airway, prevent complications due to intubation, clear airway secretions easily, and to facilitate the patient's speech and oral nutrition with this process (46). There are various methods in PDT applications. In one of these, Griggs technique; the cannula is placed in the trachea by performing tracheal dilatation with specially designed forceps (7). The most common complications of the PDT include bleeding, hypoxia, pneumothorax, pneumomediastinum, subcutaneous emphysema, malposition, and posterior tracheal wall damage (8). Because of serious complications enough experience could be crucial to achieve successful PDT procedure. Therefore adequate residency training is a key factor to prevent PDT complications. Manikinbased and cadaver studies have been performed to provide better hand-skill (9). We think that during PDT procedure needle puncture is a stressful period due to the risk of damage to the posterior wall of the trachea and nearby vascular structures and short needle length could be safer in PDT intervention.

Fiberoptic bronchoscopy (FOB) intervention helps the safe and easy application of PDT by providing vision 
inside the trachea. There are studies indicating that the FOB reduces complications such as pneumothorax, paratracheal placement of the tracheostomy cannula, posterior tracheal wall damage $(10,11)$. The minitracheostomy is an intervention that performed easily and frequently in emergency services in case of emergent airway management. It is also used to facilitate secretion cleaning and reduce FOB requirements (12). Prophylactic use of mini-tracheostomy is also recommended in patients with high risk for secretion retention $(12,13)$.

The mini-tracheostomy is applied through the cricothyroid membrane in clinical practice. It is also performed through the subcricoid region. This application provides some advantages such as less development of stenosis, and if needed standard percutaneous tracheostomy can be easily performed through this tract (14). Mini-tracheostomy needles are short and the tip of needle is curved. These properties of needle provide some advantages particularly to prevent complications. Although we couldn't find any data about the use of mini-tracheostomy needle in PDT applications in the literature, using mini-tracheostomy needle during PDT training might be safer for residents with fewer experiences.

We hypothesized that, the use of a mini-tracheostomy needle during PDT may be appropriate, especially for ICU residents' training program in order to minimize serious complications such as the posterior tracheal wall damage that may develop during the procedure. In addition, we considered performing the procedure with the aid of FOB will be effective in reducing complications.

In this descriptive study, we aimed to evaluate the bedside PDTs, performed with FOB-guided Griggs technique using a mini-tracheostomy needle, by residents in ICU patients retrospectively.

\section{MATERIAL AND METHOD}

The study was carried out with the permission of the Ethics Committee of Ankara Atatürk Chest Diseases and Chest Surgery Training and Research Hospital Clinical Researchs Ethics Committee (permission date: 14/01/2021, decision number: 709). All procedures were performed adhered to the ethical rules and the Helsinki Declaration of Principles.

The PDTs performed in 20 ICU patients using a minitracheostomy needle (Minitrach ${ }^{\oplus}$ II, Portex, UK) (Figure 1) were examined. Demographic data, duration and number of intubation, the Glasgow Coma Scale (GCS), Acute Physiology and Chronic Health Assessment
(APACHE) II, Sepsis-Related Organ Failure Assessment (SOFA) scores of the patients who were clinically suitable for PDT, duration of the procedure, those who switched to home-type MV, and those transferred from the ICU to the palliative care unit, procedure-related complications, and 90 -day mortality were recorded retrospectively.

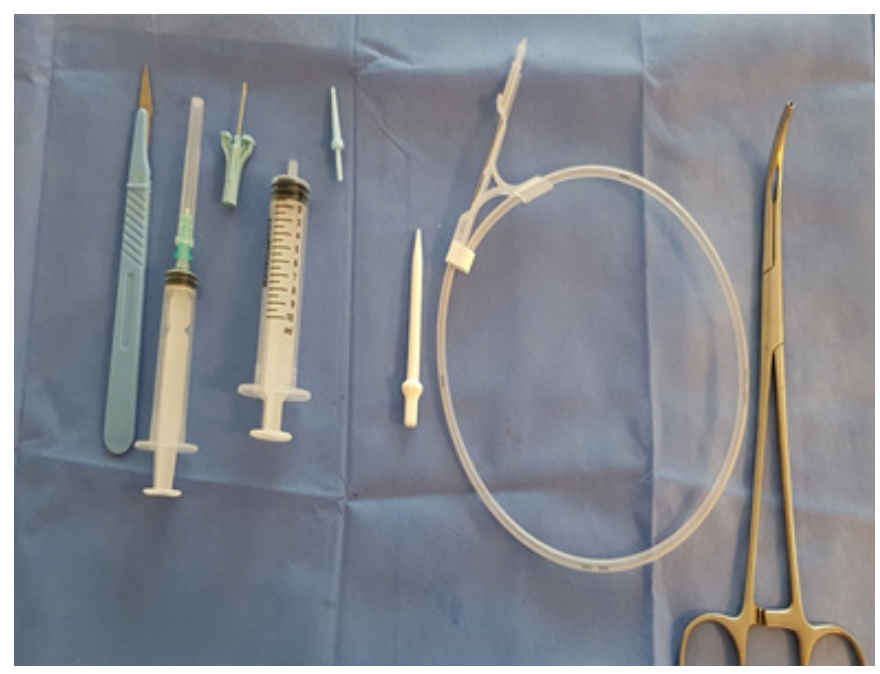

Figure 1. Mini-trach needle

\section{Protocol}

All patients were prepared for tracheostomy in accordance with the standard protocol used in our clinic. Patients were given intravenously $1 \mathrm{mcg} / \mathrm{kg}$ fentanyl, $0.5-1 \mathrm{mg} / \mathrm{kg}$ propofol, and $0.5 \mathrm{mg} / \mathrm{kg}$ rocuronium before the procedure. Five minutes before the procedure, the fraction of inspired oxygen ( $\mathrm{FiO} 2)$ of $100 \%$ and respiratory rate of $20 / \mathrm{min}$ were set in the controlled mechanical ventilation mode.

During the procedure, all patients were followed up with electrocardiogram, peripheral oxygen saturation and invasive arterial pressure monitoring.

A roller blanchett was placed under the shoulder of the patient with head in extension position to increase the view of the tracheostomy area. During the procedure, the eyelids were closed to protect the eyes. In sterile conditions, the application area was cleaned with povidin iodine and covered. Local anesthesia was applied with 3 $\mathrm{ml}$ of $2 \%$ lidocaine.

All PDT procedures were performed by a resident who was currently undergoing ICU residency training and had at least 10 PDT experience, in the presence of an experienced anesthesiologist.

During the procedure, an ICU nurse was also included in the team. Griggs technique was used in all procedures (7). A catheter mount was attached to the endotracheal tube to allow both passage of the bronchoscope and simultaneous ventilation. 
The endotracheal tube was withdrawn from the trachea after determining the translumination effect of the light of the bronchoscope on the skin. It was entered with a 2 $\mathrm{cm}$ long, 16 Gauge and beveled Mini-trach ${ }^{\odot}$ needle and advanced until air was aspirated into the injector (Figure 2). By seeing the tip of the needle on the screen of the bronchoscope, the guidewire was advanced through Mini-trach ${ }^{\oplus}$ needle (Figure 3). The skin and trachea were enlarged with forceps after dilated with an 8 French dilator (Portex ${ }^{\oplus}$, Percutanous Dilation Tracheostomy Kit, $\mathrm{UK}$ ), then an appropriate size of tracheostomy cannula was inserted over the guidewire. All patients' chest X-Ray were evaluated after procedure to rule out complications. Complications such as minor and major bleeding during and after the procedure, subcutaneous emphysema, pneumothorax, posterior tracheal wall injury, tracheal tube misplacement, and cuff puncture were recorded. Moreover, the time from needle insertion to the insertion of the tracheostomy cannula was also noted.

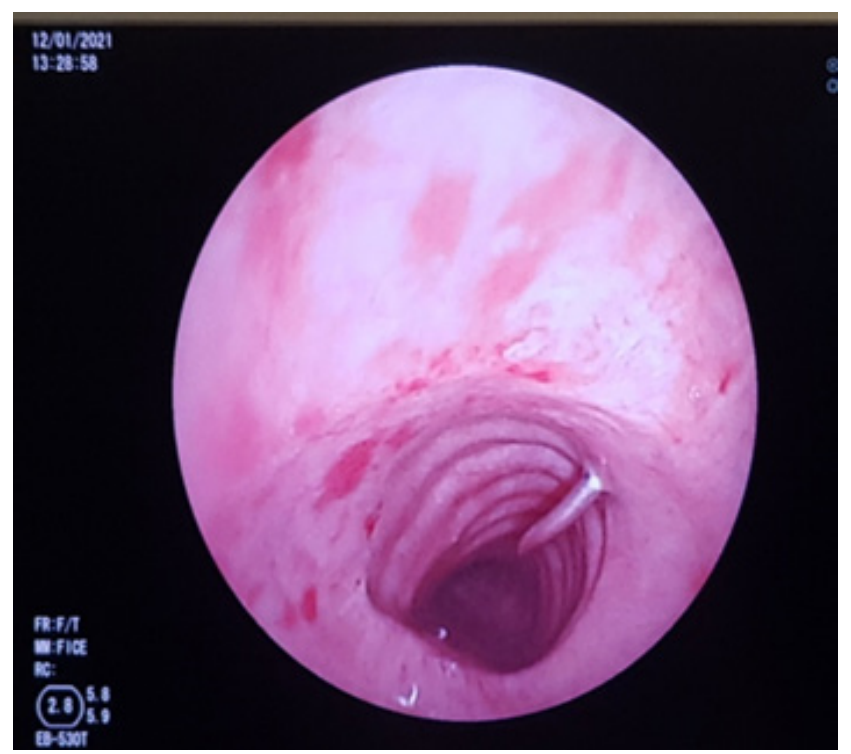

Figure 2. The view of entry with mini-trach needle

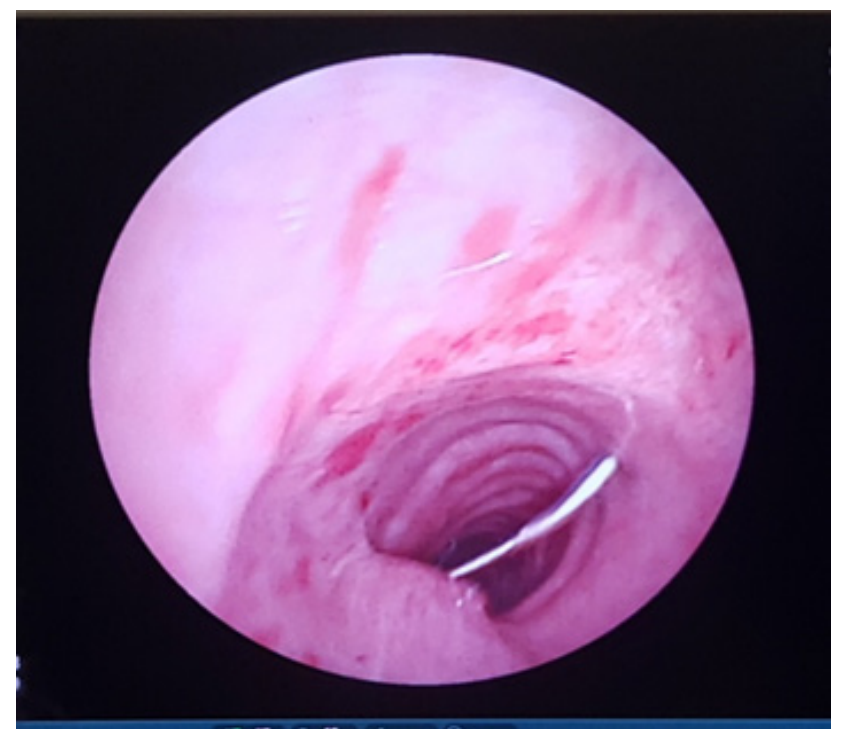

Figure 3. The view of guide wire passing through the needle

\section{Statistical Analysis}

Descriptive statistics of the data were presented as mean \pm standard deviation for continuous variables, and as number and (\%) for categorical variables.

\section{RESULTS}

Data of 20 ICU patients who underwent PDT using Minitrach $^{\otimes}$ needle with FOB guidance were retrospectively evaluated. 8 of them were female (40\%) and $12(60 \%)$ were male. The average age of the patients was $69.8 \pm 16.14$ years. Respiratory failure was present in 13 patients (65\%) due to chronic obstructive pulmonary disease, 3 patients (15\%) due to malignancy, and $4(20 \%)$ patients due to neurological diseases. The mean APACHE II score of the patients was $23.05 \pm 6.16$, GCS score was $10 \pm 3.43$, and SOFA score was 7.2 \pm 2.11 (Table 1).

The mean procedure time was $13 \pm 1.68$ minutes, and the day of tracheostomy application was $6.35 \pm 4.59$. Twelve (60\%) patients were switched to home-type ventilators. Ten (50\%) of the patients were transferred to the palliative ward with home-type ventilator. Mortality rate was $20 \%$ (4 patients) at 90 days (Table 2).

\section{Table 1. Demographic data and comorbidities of the patients}

\begin{tabular}{|lcc|}
\hline Variables & n (20)-\% & Mean \pm sd \\
\hline Gender (female/male) & $8-40 / 12-60$ & - \\
Age (year) & - & $69.8 \pm 16.14$ \\
BMI $\left(\mathrm{kg} / \mathrm{m}^{2}\right)$ & - & $25.05 \pm 2.19$ \\
APACHE II score & - & $23.05 \pm 6.16$ \\
SOFA score & - & $7.2 \pm 2.11$ \\
GCS score & - & $10 \pm 3.43$ \\
COPD & $13-65$ & - \\
Malignancy & $3-15$ & - \\
Neurological diseases & $4-20$ & - \\
\hline $\begin{array}{l}\text { Variables are given as number-percentage or mean } \pm \text { standard deviation. BMI: Body } \\
\text { mass index, GCS: Glasgow coma scale, APACHE II: Acute physiology and chronic } \\
\text { health evaluation II score, SOFA: Sepsis-related organ failure assessment score. } \\
\text { COPD:Chronic obstructive pulmonary disease }\end{array}$ & \multicolumn{2}{l}{} \\
\hline
\end{tabular}

Table 2. Percutaneous dilatational tracheostomy procedure duration, tracheostomy application day and intensive care outcomes of the patients

\begin{tabular}{|c|c|c|}
\hline Variables & n (20)-\% & Mean \pm sd \\
\hline Procedure time $(\mathrm{min})$ & - & $13 \pm 1.68$ \\
\hline $\begin{array}{l}\text { Time until tracheostomy procedure } \\
\text { (day) }\end{array}$ & - & $6.6 \pm 4.59$ \\
\hline $\begin{array}{l}\text { Number of patients switching to } \\
\text { home-type MV }\end{array}$ & $12-60$ & - \\
\hline 90-day mortality & $4-20$ & - \\
\hline
\end{tabular}

In patients evaluation before the tracheostomy application, it was observed that, extubation was tried once in 3 patients and twice in $4(20 \%)$ patients, while extubation could not be performed at all in 13 patients $(65 \%)$. When patients were evaluated in terms of 
complications; none of the patients had pneumothorax, subcutaneous emphysema, posterior tracheal wall damage, or tracheoesophageal fistula. Minimal bleeding that required no intervention was observed in only one patient.

\section{DISCUSSION}

The results of the present study showed that, there were no serious complication in PDTs performed using Minitrach $^{\circledast}$ needle by ICU residents with little experience in PDT procedure. We did not encounter any complications other than minimal bleeding in a patients who underwent PDT with this technique. Performing the PDTs with the FOB-guided Griggs technique may have contributed to procedure be faster and safer.

The PDT is one of the invasive methods commonly applied to critically ill patients in the ICU. Conditions such as the need for airway protection and avoiding longterm invasive mechanical ventilation are among its main indications (15).

There is no definitive protocol for its indications, timing, and methods. A comprehensive evaluation and teamwork are key to success in preventing procedural complications. The PDT in ICU is usually performed by two clinicians, at least one of which is experienced, and an ICU nurse.

The PDT application is one of the important intervention during ICU residency training. It is stated that, in PDT technique, familiarity with the process can be achieved after 5-10 procedures, but at least 20 PDT experiences are required to be familiar with possible complications and risks (9). All PDT training procedures are performed using a regular needle but in this study we performed this procedure by using mini-tracheostomy needle. The PDT procedures were applied by two intensive care physicians, at least one of which is experienced. The resident who performed the procedure, under the supervision of an experienced ICU doctor, had at least 10 PDT experiences. We think that mini-tracheostomy needle could provide safe procedure during residency training because of the short needle length.

Application of minitracheostomy from the subcricoid area has many advantages over the one performed through the cricothyroid membrane. Some of these advantages are; the procedure can be performed percutaneously in the intubated patient, it does not cause subglottic stenosis and voice change. In addition, application of PDT through this stoma could be easier and safer (14). Since the PDT needle is a plastic-sheathed needle, in our experience while plastic sheath is pushed towards the trachea after the needle is pulled-back, it may be bend in some interventions. However, the tip of the metal Mini-trach ${ }^{\circledR}$ needle is blunt, and curved with no tendency of bending or breaking. In addition, damage to the posterior wall of trachea could be technically rare, as its length is shorter than the standart PDT needle. In all cases we performed with the aid of FOB, the Mini-trach ${ }^{\oplus}$ needle remained in the trachea at a fairly safe distance from the posterior wall and the plastic wings of the needle prevented further advancement.

The most common complications of PDT include bleeding, subcutaneous emphysema, malposition, and posterior tracheal wall damage. Hazard et al. (16) evaluated bleeding and subcutaneous emphysema as minor complications of PDT, and reported that their incidence was low. In the same study, it was observed that the complications of minitracheostomy performed from subcricoid localization were less than PDT.

In cases of tracheostomy examined prospectively between 2005 and 2007, by Yeniaras et al. (8) it was found that, 4 (3.5\%) cases had moderate bleeding, 1 case $(0.8 \%)$ delayed bleeding, 3 cases (2.6\%) stoma infection, and 1 case $(0.8 \%)$ had subcutaneous emphysema due to malposition of the tracheostomy cannula. In present study, Mini-trach ${ }^{\circledR}$ needle was used in all patients, no complications were encountered during the procedure and at the early stage, except for minimal bleeding that developed in only one patient.

In a study comparing QuickTrach ${ }^{\text {tix }}$ (for cricothyrotomy) with Melker ${ }^{\mathrm{TN}}$ kit (for percutaneous cricothyrotomy) in a patient simulation manikin; both cricothyrotomy and percutaneous cricothyrotomy have been evaluated as easy and rapid techniques with high success rates (17). According to the results of our study, PDTs performed by clinicians during the training process might have less complication rates with the Mini-trach ${ }^{\circ}$ needle in combination with PDT set. However, we think that comparative studies are needed in larger study groups. Our study had some limitations. The study was planned retrospectively and there is no comparison group. Since the study was conducted with a specialized procedure, the number of cases was low, and this situation has significantly limited our capacity to identify the complications rate and deficiencies accurately.

\section{CONCLUSION}

There was no serious complication in PDTs performed using Mini-trach ${ }^{\circledast}$ needle in combination with the PDT set, by ICU residents with little experience other than minimal bleeding in a patient. Using Mini-trach ${ }^{\oplus}$ needle in PDTs performed via FOB by less experienced residents may be safer to prevent complications. On the other hand, more extensive and comparative studies are needed on this subject. 


\section{ETHICAL DECLARATIONS}

Ethics Committee Approval: The study was carried out with the permission of the Ankara Atatürk Chest Diseases and Chest Surgery Training and Research Hospital Clinical Researchs Ethics Committee (permission date: 14/01/2021, decision number: 709).

Informed Consent: Because the study was designed retrospectively, no written informed consent form was obtained from patients.

Referee Evaluation Process: Externally peer-reviewed.

Conflict of Interest Statement: The authors have no conflicts of interest to declare.

Financial Disclosure: The authors declared that this study has received no financial support.

Author Contributions: All of the authors declare that they have all participated in the design, execution, and analysis of the paper, and that they have approved the final version.

\section{REFERENCES}

1. Kumar M, Trikha A, Chandralekha. Percutaneous dilatational tracheostomy: Griggs guide wire dilating forceps technique versus ULTRA-perc single-stage dilator- A prospective randomized study. Indian J Crit Care Med 2012; 16: 87-92.

2. Griggs WM, Myburgh JA, Worthley LIG. A prospective comparison of a percutaneous tracheostomy technique with standard surgical tracheostomy. Intensive Care Med 1991; 17: 261-3.

3. Angel LF, Simpson CB. Comparison of surgical and percutaneous dilatational tracheostomy. Clin Chest Med 2003; 24: 423-9.

4. Duger C, Isbir AC, Uysal IO, Kol IO, Kaygusuz K, Gürsoy S. Evaluation of surgical and percutaneous tracheostomies performed in intensive care unit in terms of complications. Turk J Anaesth Reanim 2013; 41: 84-7.

5. Pappas S, Maragoudakis P, Vlastarakos P, et al. Surgical versus percutaneous tracheostomy: an evidence-based approach. Eur Arch Otorhinolaryngol 2011; 268: 323-30.

6. Sagıroglu AE, Agkoc E, Dogan Y, Gömeçlioğlu V, Orhon Z, Yavaş C. Comparison of percutaneous and surgical tracheostomy in the intensive care unit. Goztepe Medical J 2010; 25: 67-70.

7. Griggs WM, Worthley LI, Gilligan J, Thomas PD, Myburg JA. A simple percutaneous tracheostomy technique. Surg Gynecol Obstet 1990; 170: 543-5.

8. Yeniaras E, Teomete GC, Karabakh G, Topuz C, Toprak N, Ozyuvac1 E. Our Percutaneous Dilatational Tracheostomy Experience. Istanbul Medical J 2007: 4; 49-527-12.

9. Massick DD, Powell DM, Price PD, et al. Quantification of the learning curve for percutaneous dilatational tracheotomy. Laryngoscope 2000; 110: 222-8.

10.Hinerman R, Alvarez F, Keller CA. Outcome of bedside percutaneous tracheostomy with bronchoscopic guidance. Intensive Care Med 2000; 26: 1850-6.

11. Reilly PM, Sing RF, Giberson FA. Hypercarbia during tracheostomy: A comparison of ercutaneous, endoscopic, percutaneous Doppler, and standard surgical tracheostomy. Intensive Care Med 1997; 23: 859-64.
12. Wright CD. Minitracheostomy. Clin Chest Med 2003; 24: 431-5.

13. Abdelaziz M, Naidu B, Agostini P. Is prophylactic minitracheostomy beneficial in high-risk patients undergoing thoracotomy and lung resection? Interact Cardiovasc Thorac Surg 2011; $12: 615-8$.

14. Van Heurn, LW Ernest, van Geffen GJ, Brink PRG. Percutaneous subcricoid minitracheostomy: report of 50 procedures. Ann Thorac Surg 1995, 59.3: 707-9.

15. Raimondi N, Vidal Macarena R, Calleja J, et al. Evidence-based guidelines for the use of tracheostomy in critically Ill patients. J Crit Care 2017; 38: 304-18.

16. Hazard P, Jones C, Benitone J. Comparative clinical trial of standard operative tracheostomy with percutaneous tracheostomy. Crit Care Med 1991; 19: 1018-24.

17. Buonopane CE, Pasta V, Sottile D, et al. Cricothyrotomy performed with the Melker ${ }^{\text {rut }}$ set or the QuickTrach ${ }^{\text {tw }}$ kit: procedure $^{2}$ times, learning curves and operators' preference. G Chir 2014; 35: 165-70. 